\title{
Using the T-scan III System to Analyze Occlusal Function in Mandibular Reconstruction Patients: A Pilot Study
}

\author{
Chao-Wei Liu ${ }^{1}$, Yang-Ming Chang ${ }^{2}$, Yu-Fu Shen ${ }^{1}$, Hsiang-Hsi Hong ${ }^{3}$
}

Background: This study was designed to analyze the post-rehabilitation occlusal function of subjects treated with complex mandibular resection and subsequently rehabilitated with fibula osteoseptocutaneous flaps, dental implants, and fixed prostheses utilizing the T-scan system.

Methods: Ten mandibular complex resection cases that adopted fibula osteoseptocutaneous flaps, dental implants, and fixed prostheses to reconstruct occlusal function were analyzed. The mandibular reconstructions were divided into three groups based on size: full mandibular reconstructions, mandibular reconstructions larger than half of the arch, and mandibular reconstructions smaller than half of the arch. The T-scan III system was used to measure maximum occlusal force, occlusal time, anterior-posterior as well as left-right occlusal force asymmetries, and anterior-posterior as well as left-right asymmetrical locations of occlusal centers.

Results: $\quad$ Subjects with larger mandibular reconstructions and dental implants with fixed partial dentures demonstrated decreased average occlusal force; however, the difference did not reach the statistically significant level $(p>0.05)$.

At a Glance Commentary

Scientific background of the subject

Mandibular osseous reconstructions are difficult to implement and often fail to satisfy the patients' expectations for oropharyngeal function and esthetics. Also, there are several methods including a computer-aided video system, a photo-occlusion method, and the T-scan system available to evaluate occlusal function in the clinical setting.

\section{What this study adds to the field}

This study used the T-scan III system to analyze the post-rehabilitation occlusal function of subjects treated with complex mandibular resection and subsequently rehabilitated with fibula osteoseptocutaneous flaps, dental implants, and fixed prostheses.

The most significant asymmetry of occlusal center location occurred among subjects with mandibular reconstructed areas larger than half of the mandibular arch.

Conclusions: Comparison of the parameters of T-scan system used to analyze the occlusal function showed that the occlusal force was not an objective reference. Measurements of the location of the occlusal center appeared more repeatable, and were less affected by additional factors. The research results of this study showed that the size of a reconstruction did not affect the occlusal force after reconstruction and larger reconstructed areas did not decrease the average occlusal force. The most significant parameter was left and right asymmetry of the occlusion center (LROC) and was measured in subjects with reconstruction areas larger than half of the arch.

(Biomed J 2015;38:52-57)

Key words: dental implants, mandibular reconstruction, T-scan system

$\mathrm{T}$ The mandible is an essential facial structure and a primary component in masticatory function. The structure of the

mandibular complex includes the tongue, mandible, and the soft tissue surrounding the mandible. When a malignant

\footnotetext{
From the ${ }^{1}$ Department of Prosthodontics, Chang Gung Memorial Hospital at Linkou, Chang Gung University College of Medicine, Taoyuan, Taiwan; ${ }^{2}$ Department of Oral and Maxillofacial Surgery, Chang Gung Memorial Hospital at Taipei, Chang Gung University College of Medicine, Taoyuan, Taiwan; ${ }^{3}$ Department of Periodontics, Chang Gung Memorial Hospital at Linkou, Chang Gung University College of Medicine, Taoyuan, Taiwan

Received: Jan. 17, 2013; Accepted: Feb. 24, 2014

Correspondence to: Dr. Yang-Ming Chang, Department of Oral and Maxillofacial Surgery, Chang Gung Memorial Hospital at Taipei. 199, Dunhua N. Rd., Taipei 105, Taiwan (ROC). Tel: 886-2-27135211 ext. 3493; Fax: 886-2-25148246; E-mail: c430502@cloud.cgmh.org.tw
}

DOI: $10.4103 / 2319-4170.128722$ 
mandibular lesion is diagnosed, a complex mandibular resection must be performed to ensure that tumor cells in the lesions are removed. Extensive mandibular complex resections will alter patient's appearance and degrade the oropharyngeal function. In addition, extensive mandibular resections result in oral environments that are problematic for prosthetic reconstruction, such as reduced alveolar bone, loss of continuity between the two sides of the mandible, intraoral soft tissue defects, insufficient vestibular depth due to excessive scar tension at the surgical sites, as well as insufficient salivary secretion, and sensory and movement malfunctions after tongue and lip surgeries.

Mandibular osseous reconstructions which adopt dental prostheses are difficult to implement and often fail to satisfy the patients' expectations for oropharyngeal function and esthetics. According to the clinical literature, ${ }^{[1-3]}$ the methods most commonly utilized in reconstructing occlusal function following mandibular complex resections include the use of fibula osteoseptocutaneous flaps to repair defective tissue and dental implants with fixed prostheses to restore occlusal function. Compared to the flap grafting of other body parts, fibula osteoseptocutaneous flaps are flexible, small, and beneficial for intraoral reconstruction. The success rate of dental implants is improved because the outer fibular cortical bone is more stable. ${ }^{[1]}$

There are several methods available to evaluate occlusal function in the clinical setting, but all lack evidence-based support to verify quantitative data evaluation. The commonly used quantitative occlusal approaches include a computer-aided video system, a photo-occlusion method, and the T-scan system. ${ }^{[4-6]}$

The T-scan system utilizes a 0.1 -mm-thick sensor made with a flexible material that can prevent errors of mandibular deviation caused by excessively thick or hard sensors when occluding. The parameters of the T-scan III system, including introduction time, occlusal center, track of occlusal center, and percentage of occlusal force distribution, can provide a reference for analyzing instant occlusal conditions. These parameters can also form a time track distribution of the points of maximum occlusion and occlusal force through contact of the teeth with the sensor. This information is beneficial for quantitative research in occlusal equilibrium. The T-scan III system sensor comprises two polyester films as substrate, conductive ink as an interlayer, and a vertical and horizontal woven wire grid with approximately 1500 sensor points. Since polyester films have high tear and strain resistance, the thin polyester films in the T-scan III system can endure occlusal force and change form during occlusal movement. The sensor is approximately $60 \mu \mathrm{m}$ thick, which does not hinder the subjects when conducting different types of occlusal exercises. When occlusal force is applied to the sensor, the sensor points change local electronic resistance due to stress. The system measures the changes in current loops. After collecting the data, corresponding software can be utilized to conduct quantitative analysis of the changes in occlusal contact points and force over time and then compute a distribution of the occlusal forces. Compared to previous systems, the T-scan III system has improved accuracy and repeatability. In measuring force distribution, the system can analyze the percentage of force distribution of one tooth, the anterior and posterior occlusal forces, and the left and right occlusal forces. Regarding occlusal center, the system can record the track of occlusal center from the initial contact position to the final position. ${ }^{[7]}$

Currently in clinical researches, the T-scan system has been utilized to analyze malocclusion among temporomandibular disorder (TMD) patients and occlusal function among orthodontic patients before and after treatment. This is the first study designed to analyze the post-rehabilitation occlusal function of subjects treated with complex mandibular resection and subsequently rehabilitated with fibula osteoseptocutaneous flaps, dental implants, and fixed prostheses.

\section{METHODS}

Adult male and female subjects from the Chang Gung Memorial Hospital were enrolled into the study based upon the following criteria:

- $\quad$ Subjects with composite mandibular defects after surgical treatment of mandibular tumor underwent reconstruction with a free fibula osteoseptocutaneous flap combined with dental implants and implant-supported prosthesis.

- Subjects needed to be available for the duration of the study and had to sign an informed consent form.

- Subjects were regularly monitored with panoramic radiographs to check for tumor recurrence and to monitor bone loss and inflammation around the implants.

- $\quad$ Subjects were excluded from the study if they had recurrent lesions or had received postoperative radiotherapy.

Ten patients diagnosed with mandibular tumors were treated with mandibular complex resections and subsequent fibula osteoseptocutaneous flaps, simultaneously implanting dental implants (3i system; Implant Innovations, Inc., Palm Beach Gardens, FL, USA) in the fibular bones and the rest of the mandible. These procedures were completed from July 1999 to April 2007 [Table 1]. After 4-8 months, fixed prostheses were adopted to restore occlusal function. Fixed prostheses were splinted to build multi-unit fixed bridges. After an average trace period of 7.38 years following the mandibular resections, alveolar bone resorption around the implants was examined with intraoral radiography. In addition, the T-scan III system was used to measure occlusal 
function by measuring the occlusal records during maximum occlusal contact. The study protocol was approved by the Institutional Review Board (IRB) of Chang Gung Memorial Hospital and the permit number of IRB was 101-1364B.

The components of a T-scan III system (Tekscan, Inc., Boston, MA, USA) are listed below [Figure 1]:
a. Scanning handle
b. Sensor $(60 \mu \mathrm{m})$
c. Sensor support.

During data collection, the subjects were seated in the same position. Before conducting the experiment, each subject practiced moving their mandible from the rest position to the intercuspal position (ICP) using a comfortable amount of biting pressure. After the subjects were familiar with the procedure, the operator put the sensor support into their mouths and recorded the contact conditions when occluding. The sensor was set on the handle in advance and was positioned close to the maxillary occlusal plane. The subjects were then instructed to close their mouths in the ICP. The IP model of the T-scan III system was used to record the process as the mandible moved from the rest position to the ICP and then back to the rest position. Each subject repeated this procedure three times and the results were recorded.

The T-scan III system recorded the changes in occlusal contact at a time interval of $0.01 \mathrm{~s}$ producing dynamic

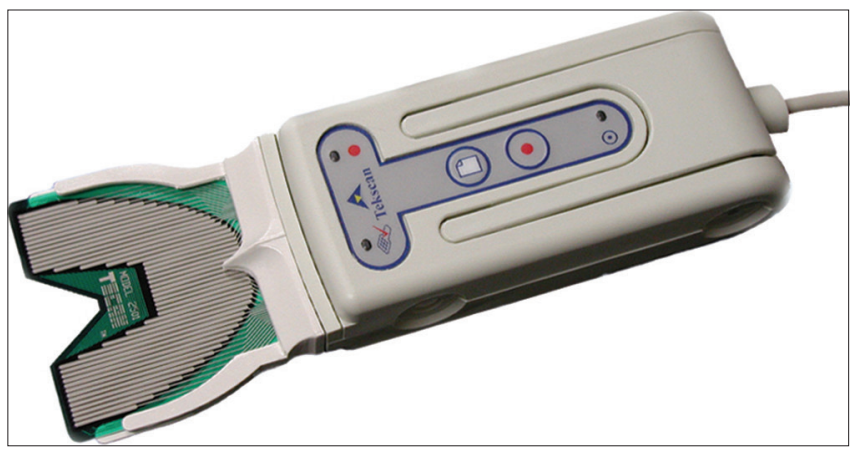

Figure 1: T-scan III system. change data. The recorded data included contact locations and relative occlusive forces. The IP model of the T-scan III system was selected to estimate the time required for occluding in the ICP, measuring the time for completing the occlusal movement from the resting position to the ICP, measuring the anterior and posterior as well as the left and right asymmetrical locations of the occlusal centers, and the asymmetries of occlusal force [Figure 2]. The following equations were used to calculate occlusal force asymmetry:

Anterior-posterior asymmetry (\%): (total anterior occlusal force - total posterior occlusal force)/(total anterior occlusal force + total posterior occlusal force) $\times 100 \%$

Left-right asymmetry: (total right occlusal force - total left occlusal force)/(total right occlusal force + total left occlusal force) $\times 100 \%$

The 10 subjects were classified into three groups based on the size of the prosthetic reconstructions following mandibular resections as follows: Full mandibular reconstruction (Group 1) [Figure 3], mandibular reconstruction larger than half of the arch (Group 2) [Figure 4], and mandibular reconstruction less than half of the arch (Group 3) [Figure 5].

In order to analyze the statistical difference of the average data among these three groups, Kruskal-Wallis test was used. In addition, the Mann-Whitney test was used to test the statistical difference between groups.

\section{RESULTS}

Among the 10 subjects in the study, 6 were males and 4 were females; their average age was 50.75 (45-60) years. The subjects received a total of 41 implants. The average trace period of the implants was 49.25 months, and the survival rate of the implants reached $100 \%$. After clinical and radiographic examinations, none of the 10 subjects demonstrated recurrent lesions, osteoseptocutaneous flap failures, osseointegration degradation of the implants, or dental prostheses failure. The survival rate of the 41 implants was 100\% during an average trace period of 49.25 months. The minimum and maximum trace periods were 21 and 141 months, respectively.

Table 1: Ten mandibular complex resection cases

\begin{tabular}{|c|c|c|c|c|c|c|c|c|c|c|c|}
\hline Case & Group & $\begin{array}{l}\text { Age } \\
\text { (years) }\end{array}$ & Gender & Disease & $\begin{array}{l}\text { Resected part } \\
\text { of the mandible }\end{array}$ & $\begin{array}{c}\text { Dental } \\
\text { implants }\end{array}$ & $\begin{array}{l}\text { Implant survival } \\
\text { (numbers) }\end{array}$ & $\begin{array}{l}\text { Unit of } \\
\text { prosthesis }\end{array}$ & $\begin{array}{c}\text { Follow-up } \\
\text { (months) }\end{array}$ & $\begin{array}{c}\text { Marginal bone } \\
\text { loss }(\mathrm{mm})\end{array}$ & $\begin{array}{c}\text { Date of } \\
\text { inspection }\end{array}$ \\
\hline 1 & 1 & 45 & F & A & $\mathrm{F}$ & 5 & 5 & 10 & 23 & $<1.0$ & $19 / 07 / 2012$ \\
\hline 2 & 1 & 62 & M & A & $\mathrm{F}$ & 4 & 4 & 12 & 73 & $<1.0$ & $11 / 09 / 2012$ \\
\hline 3 & 1 & 54 & M & A & $\mathrm{F}$ & 6 & 6 & 13 & 144 & $<1.5$ & 03/05/2012 \\
\hline 4 & 2 & 60 & M & A & $\mathrm{LA}+\mathrm{LL}$ & 5 & 5 & 11 & 110 & $<1.0$ & $23 / 07 / 2012$ \\
\hline 5 & 2 & 47 & F & A & $\mathrm{LA}+\mathrm{LR}$ & 4 & 4 & 9 & 78 & $<1.0$ & $28 / 08 / 2012$ \\
\hline 6 & 2 & 57 & M & SCC & $\mathrm{LA}+\mathrm{LR}$ & 3 & 3 & 5 & 49 & $<1.5$ & $10 / 08 / 2012$ \\
\hline 7 & 2 & 46 & M & A & $\mathrm{LA}+\mathrm{LR}$ & 4 & 4 & 10 & 142 & $<1.0$ & $04 / 10 / 2012$ \\
\hline 8 & 3 & 45 & M & SCC & LR & 3 & 3 & 6 & 96 & $<1.0$ & $04 / 10 / 2012$ \\
\hline 9 & 3 & 46 & F & A & LL & 2 & 2 & 3 & 70 & $<1.0$ & $17 / 09 / 2012$ \\
\hline 10 & 3 & 38 & F & A & LA & 5 & 5 & 13 & 66 & $<1.0$ & $25 / 09 / 2012$ \\
\hline
\end{tabular}

Abbreviations: A: Ameloblastoma; SCC: Squamous cell carcinoma; LA: Lower arterial; LR: Lower right; LL: Lower left; F: Full mandible 


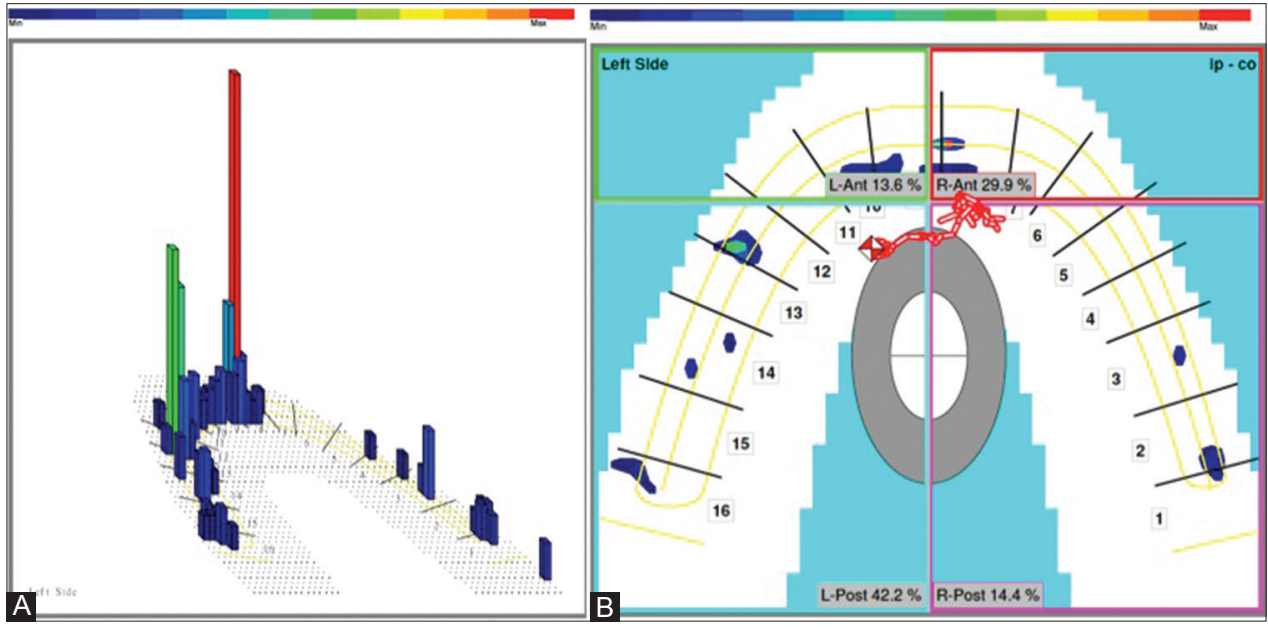

Figure 2: (A and B) Occlusal force and force distribution of the subjects in IP model using the T-scan III system.

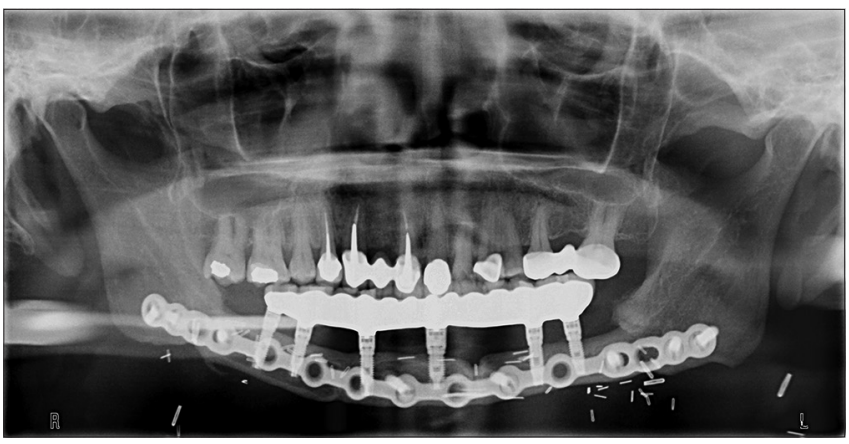

Figure 3: Full mandibular reconstruction using fibula osteoseptocutaneous flaps, dental implants, and fixed dental prostheses (Case No. 1).

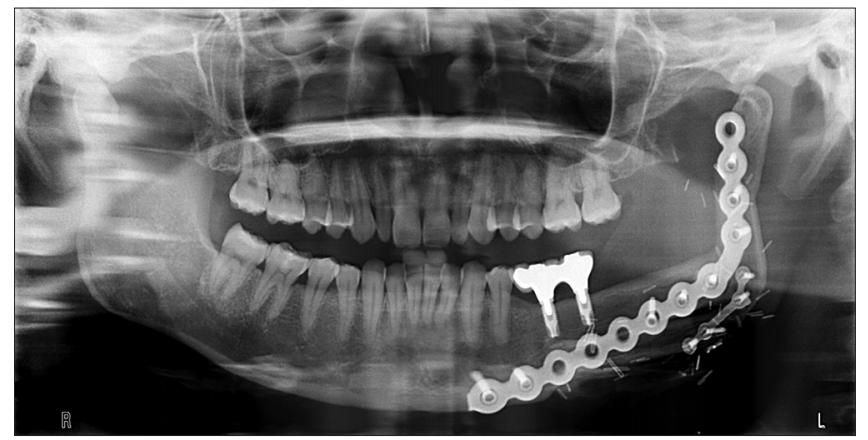

Figure 5: Mandibular reconstruction area less than half of the arch (Case No. 9).

Results of the maximum occlusal force, occlusal time, anterior-posterior and left-right asymmetrical distribution of occlusal force, and anterior-posterior and left-right locations of asymmetrical occlusal centers are presented in Table 2. The maximum occlusal force of each group was $1669.95 \mathrm{~g} / \mathrm{cm}^{2}, 1322.17 \mathrm{~g} / \mathrm{cm}^{2}$, and $2563.67 \mathrm{~g} / \mathrm{cm}^{2}$, respectively. The average time to finish one occlusion to the ICP of each group was $2.94 \mathrm{~s}, 1.93 \mathrm{~s}$, and $2.25 \mathrm{~s}$, respectively. The left-right asymmetries in occlusal force were $28.39 \%$,

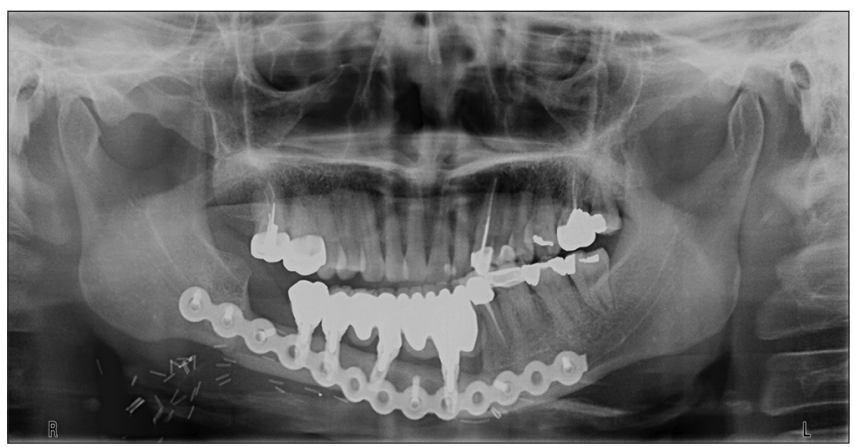

Figure 4: Mandibular reconstruction area larger than half of the arch (Case No. 5).

$67.20 \%$, and $22.53 \%$, respectively, and the anterior-posterior asymmetries in occlusal force were $35.75 \%, 65.17 \%$, and $66.30 \%$, respectively, in the three groups. The location of the anterior-posterior asymmetrical occlusal centers was $5.45 \mathrm{~mm}, 12.13 \mathrm{~mm}$, and $14.22 \mathrm{~mm}$, respectively, and that of the left-right asymmetrical occlusal centers was $2.89 \mathrm{~mm}$, $14.21 \mathrm{~mm}$, and $8.56 \mathrm{~mm}$, respectively, in the three groups.

Kruskal-Wallis test indicated that no statistical difference was demonstrated regarding maximum occlusal force, occlusal time, anterior-posterior occlusal force asymmetry, anterior-posterior asymmetrical location of occlusal centers, and left-right occlusal force asymmetry $(p>0.05)$. Statistical significance was observed only in the left-right asymmetrical location of occlusal centers $(p<0.05)$ [Table 2]. Mann-Whitney test revealed that groups 1 and 2 demonstrated statistical significance regarding the asymmetrical location of the occlusal centers [Table 3].

\section{DISCUSSION}

The sensitivity of the T-scan III system in measuring occlusal force has been disputed in clinical research. A number of researchers have indicated that the T-scan III system 
Table 2: Results of occlusion parameters

\begin{tabular}{|c|c|c|c|c|c|c|}
\hline & \multicolumn{2}{|c|}{$\mathrm{MO}\left(\mathrm{g} / \mathrm{cm}^{2}\right)$} & \multicolumn{2}{|c|}{ OT (s) } & \multicolumn{2}{|c|}{ APOA $(\%)$} \\
\hline & Mean \pm SD & Median (min., max.) & Mean \pm SD & Median (min., max.) & Mean \pm SD & Median (min., max.) \\
\hline 1 & $1669.95 \pm 1390.90$ & $960.67(776.67,3272.50)$ & $2.94 \pm 2.25$ & $3.90(0.705,4.37)$ & $35.75 \pm 8.60$ & $40.18(25.84,41.22)$ \\
\hline 2 & $1322.17 \pm 1284.80$ & $1054.83(85,3094)$ & $1.93 \pm 0.90$ & $2.17(1.33,3.22)$ & $65.17 \pm 38.19$ & $74.28(13.13,99.00)$ \\
\hline 3 & $2563.67 \pm 3704.45$ & $535(316.67,6839.33)$ & $2.25 \pm 0.75$ & $1.66(1.53,1.79)$ & $66.30 \pm 44.23$ & $85.60(15.71,97.60)$ \\
\hline \multirow[t]{2}{*}{$p$ value } & 0.845 & & 0.546 & & 0.588 & \\
\hline & \multicolumn{2}{|c|}{ LROA $(\%)$} & \multicolumn{2}{|c|}{$\mathrm{APOC}(\mathrm{mm})$} & \multicolumn{2}{|c|}{ LROC $(\mathrm{mm})$} \\
\hline 1 & $28.39 \pm 2.69$ & $27.38(26.36,31.43)$ & $5.45 \pm 2.80$ & $4.00(3.67,8.67)$ & $2.89 \pm 1.02$ & $2.67(2.0,4.0)$ \\
\hline 2 & $67.20 \pm 25.59$ & $64.86(39.1,100)$ & $12.13 \pm 6.63$ & $10.83(6.50,20.33)$ & $14.21 \pm 5.16$ & $14.67(7.5,20.0)$ \\
\hline 3 & $22.53 \pm 19.74$ & $16.69(6.37,44.53)$ & $14.22 \pm 8.77$ & $12.67(6.33,23.67)$ & $8.56 \pm 3.34$ & $8.33(5.3,12.0)$ \\
\hline$p$ value & 0.062 & & 0.231 & & $0.034 *$ & \\
\hline
\end{tabular}

Abbreviations: MO: Maximum occlusal force; OT: Occlusion time; APOA: Anterior and posterior occlusal asymmetry; LROA: Left and right occlusal asymmetry; APOC: Anterior and posterior asymmetry of occlusion center; LROC: Left and right asymmetry of occlusion center; ${ }^{*} p<0.05$, Kruskal-Wallis test

Table 3: Mann-Whitney test of LROC (mm)

\begin{tabular}{lcll}
\hline Group & Median (min., max.) & \multicolumn{2}{c}{$p$ value } \\
\hline 1 & $2.67(2.0,4.0)$ & & \\
2 & $14.67(7.5,20.0)$ & $0.034^{*}$ & \\
3 & $8.33(5.3,12.0)$ & $0.050^{\mathrm{a}}$ & $0.157^{\mathrm{b}}$
\end{tabular}

Abbreviation: LROC: Left and right asymmetry of occlusion center; ${ }^{*} p$ value of Group 1 compared to Group 2 and $p<0.05 ;{ }^{a} p$ value of Group 1 compared to Group $3 ;{ }^{b} p$ value of Group 2 compared to Group 3

measures the relative occlusal force of the subjects instead of the numerical values of absolute occlusal force. ${ }^{[8]}$ However, research indicates that the T-scan III system can be used as a measurement tool in clinical experiments, and the sensitivity setting can be adjusted to measure the occlusal force at different ranges. ${ }^{[4]}$ This study set the sensitivity of the T-scan III system at the low-2 level, and the measurable range was between 146 and $3821 \mathrm{~g} / \mathrm{cm}^{2}$. The average maximum occlusal force of the subjects was $1798.950 \mathrm{~g} / \mathrm{cm}^{2}$. However, the occlusal force was affected by various factors such as age ${ }^{[9]}$ sex,${ }^{[10]}$ and facial structure. ${ }^{[11]}$ Therefore, an objective reference cannot be achieved by analyzing the function of prosthetic rehabilitation through measuring the occlusal force. In this experiment, no significant between-group differences were observed regarding the occlusal force and mandibular reconstruction size.

Sierpińska et al.'s studies ${ }^{[12,13]}$ explore the relationship of occlusal time and masticatory function, and report that when the upper and lower occluding pairs of posterior teeth were decreased, the duration of every chewing cycle was shortened and the subjects needed to increase total chewing time to compensate and maintain the occlusal function. In the present study, we compared the occlusal time among the three groups and the results showed no statistical difference. Therefore, occlusal efficiency did not appear to be correlated with the size of mandibular reconstruction.

The three groups showed significant differences in the locations of the left-right asymmetrical center of occlu- sion. The mandibular reconstruction size affected the left and right levels of asymmetry. Subjects with mandibular reconstructed areas larger than half of the arch (Group 2) had the largest level of asymmetry, and the average left-right asymmetrical location of the occlusal center was $14.21 \mathrm{~mm}$. When compared with Group 2, the least asymmetrical location was observed among subjects in Group 1. The left or right side of the reconstructed area was not correlated with the left-right asymmetrical location of the occlusal center.

Using T-scan system to evaluate the normal occlusal function, the left-right occlusal center asymmetry range in $95 \%$ confidence interval is from -6.6 to $6.68 \mathrm{~mm} \cdot{ }^{\left[{ }^{[14]}\right.} \mathrm{So}$, the occlusal center asymmetry in Group 1 after reconstruction was within normal limit and in Group 2 was above normal, and there was statistical difference between them.

According to T-scan system, the occlusal center is the torque balance center of all the contact points of the teeth and every occlusal force on them. So, in this study, the occlusal center asymmetry means the occlusal torque shifting degree. The factors influencing the occlusal center include the number of contact points, the distance between each contact point and the occlusal center, and the occlusal force of every contact point. In this study, there were no statistical differences among the three groups in left and right occlusal asymmetry (LROA) and anterior and posterior occlusal asymmetry (APOA). Therefore, the reason of occlusal center asymmetry might be the left-right occlusal contact point in numerical difference.

In full mandibular reconstruction (Group 1), it seems easier to adjust the number of occlusal contact points and occlusal force by grinding the prosthesis, to avoid occlusal center asymmetry. In Group 2, the occlusion adjustment might be restricted by the remaining teeth, for example, when the remaining teeth have less occlusal contact or there is occlusal interference, and it will be more difficult to achieve the left-right occlusal torque balance. Another 
factor is that although the mandibular defects had been reconstructed, the posterior teeth in some of the cases could not be restored with 28-teeth occlusion. In Sadamori et al.'s clinical study of TMD, ${ }^{[15]}$ the unbalanced left-right occlusal torque could cause TMD and masticatory muscle pain.

Because the location of the occlusal center is not affected by age, sex, or ethnicity, ${ }^{[16]}$ and is affected by the facial and dental asymmetry, ${ }^{[17]}$ the parameters of occlusal center and its asymmetrical degree are more reliable than the occlusal force in occlusal function analysis. These parameters can be applied to evaluate denture treatments ${ }^{[18,19]}$ and oral function. ${ }^{[20]}$ The location of the occlusal center will shift when an occlusal stop is recovered by prosthetic treatment. Thus, it is suggested that analysis of the occlusal center will be useful in diagnosing and evaluating prosthetic treatment from the standpoint of occlusal support. Therefore, according to the results of this study, in Group 2 reconstruction patients, it is suggested to fabricate the prosthesis on the remaining dentition to achieve better occlusal support and avoid occlusal center asymmetry after mandibular reconstruction.

In this study, no significant differences were observed in the occlusal force and the size of the mandibular reconstruction. However, a significant difference in the reconstruction size existed between the left and right asymmetry of the occlusion center (LROC) among these three groups. Thus, the results of this study are consistent with those reported by previous studies, that is, the occlusal center and its asymmetrical degree are more reliable parameters than the occlusal force in occlusal function analysis after mandibular reconstruction.

\section{Conclusions}

Comparing the parameters of T-scan system used to analyze the occlusal function showed that the occlusal force was not an objective reference. Measurements of the location of the occlusal center appeared more repeatable, and were less affected by additional factors. The results of this study show that the size of a reconstruction did not affect the occlusal force after reconstruction and larger reconstructed areas did not decrease the average occlusal force. The most significant parameter was LROC and was measured in subjects with reconstruction areas larger than half of the arch.

\section{REFERENCES}

1. Jones NF, Monstrey S, Gambier BA. Reliability of the fibular osteocutaneous flap for mandibular reconstruction: Anatomical and surgical confirmation. Plast Reconstr Surg 1996;97:707-16.

2. Wei FC, Seah CS, Tsai YC. Fibula osteoseptocutaneous flap for reconstruction of composite mandibular defects. Plast Reconstr Surg 1994;93:294-304.
3. Hidalgo DA. Fibula free flap: A new method of mandible reconstruction. Plast Reconstr Surg 1989;84:71-9.

4. Hirano S, Okuma K, Hayakawa I. In vitro study on accuracy and repeatability of T-scan 2 system. Kokubyo Gakkai Zasshi 2002;69:194-201.

5. Millstein PL. A method to determine occlusal contact and noncontact areas: Preliminary report. J Prosthet Dent 1984;52:106-10.

6. Arcan M, Zandman F. A method for in vivo quantitative occlusal strain and stress analysis. J Biomech 1984;17:67-79.

7. Koos B, GodtA, Schille C, Goz G. Precision of an instrumentation-based method of analyzing occlusion and its resulting distribution of forces in the dental arch. J Orofac Orthop 2010;71:403-10.

8. Throckmorton GS, Rasmussen J, Caloss R. Calibration of T-Scan sensors for recording bite forces in denture patients. J Oral Rehabil 2009;36:636-43.

9. Bakke M, Holm B, Jensen BL, Michler L, Möller E. Unilateral, isometric bite force in 8-68-year-old women and men related to occlusal factors. Eur J Oral Sci 1990;98:149-58.

10. Tuxen A, Bakke M, Pinholt EM. Comparative data from young men and women on masseter muscle fibres, function and facial morphology. Arch Oral Biol 1999;44:509-17.

11. Proffit WR, Fields HW, Nixon WL. Occlusal forces in normal-and long-face adults. J Dent Res 1983;62:566-70.

12. Sierpinska T, Golebiewska M, Długosz JW. The relationship between masticatory efficiency and the state of dentition at patients with non rehabilitated partial lost of teeth. Adv Med Sci 2006;51 Suppl 1:196-9.

13. Sierpinska T, Golebiewska M, Lapuc M. The effect of mastication on occlusal parameters in healthy volunteers. Adv Med Sci 2008;53:316-20.

14. Hu ZG, Cheng H, Zheng M, Zheng ZQ, Ma SZ. Quantitative study on occlusal balance of normal occlusion in intercuspal position. Zhonghua Kou Qiang Yi Xue Za Zhi 2006;41:618-20.

15. Sadamori S, Kotani H, Abekura H, Hamada T. Quantitative analysis of occlusal force balance in intercuspal position using the Dental Prescale system in patients with temporomandibular disorders. Int Chin J Dent 2007;7:43-7.

16. Shinogaya T, Bakke M, Thomsen CE, Vilmann A, Sodeyama A, Matsumoto M. Effects of ethnicity, gender and age on clenching force and load distribution. Clin Oral Investig 2001;5:63-8.

17. Cheong, YW, Lo LJ. Facial asymmetry: Etiology, evaluation, and management. Chang Gung Med J 2011;34:341-51.

18. Olivieri F, Kang KH, Hirayama H, Maness WL. New method for analyzing complete denture occlusion using the center of force concept: A clinical report. J Prosthet Dent 1998;80:519-23.

19. Takishita M. A clinical and physiological evaluation of prosthodontic treatment by removable partial dentures with free-end saddles-a 3.5-year follow-up study. Kokubyo Gakkai Zasshi 2001;68:254-61.

20. Satoh C, Hattori Y, Ogino Y, Azuma K, Watanabe M. A study of diagnostic test for TMD based on bite force distribution. J Jpn Soc Temporomandibular Joint 1999;11:193-200. 